\title{
Beyond the Standard Model at the LHC and Beyond
}

\author{
John Ellis \\ Theoretical Physics Division, Physics Department, CERN, CH 1211 Geneva 23, Switzerland
}

\begin{abstract}
Many of the open questions beyond the Standard Model will be addressed by the LHC, including the origin of mass, supersymmetry, dark matter and the possibility of large extra dimensions. A linear $e^{+} e$ collider (LC) with sufficient centre-of-mass energy would add considerable value to the capabilities of the LHC.
\end{abstract}

Keywords: Beyond the Standard Model, Higgs, Supersymmetry, LHC, ILC

PACS: 11.15.Ex,11.30.Pb,11.30.Qc,12.10.-g,12.60.Jv,14.80.Bn

CERN-PH-TH/2007-174

\section{OPEN QUESTIONS BEYOND THE STANDARD MODEL}

There is a standard list of fundamental open questions beyond the Standard Model:

What is the origin of particle masses?

Are they indeed due to a Higgs boson, as hypothesized within the Standard Model? If so, is the Higgs boson accompanied by some other physics such as supersymmetry? If not, what replaces the Higgs boson?

Why are there so many types of matter particles?

Related to this question is the mixing of the different flavours of quarks and leptons, and the mechanism for CP violation. This matter-antimatter difference is thought to be linked to cosmological baryogenesis. However, the Standard Model cannot, by itself, explain the cosmological matter-antimatter asymmetry, adding urgency to the search for flavour and CP violation beyond the Standard Model.

Are the fundamental forces unified?

If so, in the simplest models this unification occurs only at some very high energy

$10^{16} \mathrm{GeV}$. Physics at this scale may be probed via neutrino physics, or possibly less directly at accelerators via measurements of particle masses and couplings, and looking for unification relations between them.

What is the quantum theory of gravity?

The best candidate for such a theory may be (super)string theory, which generously predicts extra space-time dimensions as well as supersymmetry, but at what energy scale?

The good news is that all of these fundamental open questions will be addressed by the LHC: its energy should be ample for resolving the problem of mass, including the questions whether there is a Higgs boson and/or supersymmetry, a dedicated experiment 
will be examining matter-antimatter differences, models of unification could be probed via measurements of sparticle masses and couplings, and string theory might be probed via supersymmetry breaking, extra dimensions or even black hole production and decay. Accordingly, most of this talk will be concerned with LHC physics, accompanied by some asides about linear collider (LC) physics.

Supersymmetry may play a role in answering most of the open questions, for example by stabilizing the scale of electroweak symmetry breaking or by aiding the unification of the gauge coupling, and it also seems to play an essential role in string theory. Therefore, I have invested many or my personal efforts in supersymmetry, and apologize for giving pride of place to it in the rest of this talk.

\section{HIGGS PHYSICS}

Several different Higgs production mechanisms will be important at the LHC, including gluon-gluon and $W W$ fusion, and production in association with gauge bosons or heavy quarks. Several decay modes will also be important, including $\gamma \gamma$, four leptons, $\tau^{+} \tau$, $W W, \bar{b} b$, etc. [1, 2]. Accordingly the search for the Higgs boson at the LHC will require the combination of many different signatures and hence excellent understanding of all components of ATLAS and CMS. As seen in Fig. 1, once both detectors have accumulated and analyzed a couple of hundred $\mathrm{pb}{ }^{1}$ of data, they may be able to start excluding certain ranges of Higgs masses at the $95 \%$ confidence level [3]. With $1 \mathrm{fb}{ }^{1}$ of analyzed data, they might be able to exclude a Standard Model Higgs boson over the entire mass range up to $1 \mathrm{TeV}$, or they might be able to establish a five- $\sigma$ signal if the Higgs boson weighs between 150 and $500 \mathrm{GeV}$. Several $\mathrm{fb}^{1}$, corresponding to several months at one tenth of the design luminosity, would be needed to discover the Higgs boson over the entire mass range.

With some luck, it may also be possible to determine the spin of the Higgs boson [4, 5, 6]. For example, if it is light and is seen to decay into $\gamma \gamma$, it cannot have spin one. If it has higher mass and is seen to decay into four leptons, their angular correlations will enable $J^{P}$ states other than $0^{+}$to be excluded with high significance. Analogous studies could be made at a LC, also using angular distibutions and the threshold behaviour for $e^{+} e \quad$ ! $Z H[7]$.

As seen in Fig. 2, the LHC may also make a first analysis of the proportionality of the Higgs couplings to particle masses [3]. If $m_{H} \quad 120 \mathrm{GeV}$, it may be able to measure the couplings to $\tau^{+} \tau, \bar{b} b, W W, Z Z$ and $\bar{t} t$ each with an accuracy $20 \%$. Much more precise measurements could be made subsequently with a LC [8].

As well as its importance for particle physics, the discovery of a Higgs boson would also be very important for cosmology. An elementary Higgs boson would have caused a phase transition in the early Universe when it was $10^{12} \mathrm{~s}$ old, and might have generated the matter in the Universe via electroweak barygenesis. Further back in the history of the Universe, a related inflaton might have expanded the Universe exponentially when it was $10^{35} \mathrm{~s}$ old. Coming back to the present, naively the Higgs boson of the Standard Model would contribute a factor $10^{56}$ too much to the present-day dark energy, apparently requiring some 'miraculously' fine-tuned cancellation. Cosmologists should be as interested as particle physicists in the dénouement of the Higgs saga. For the time 

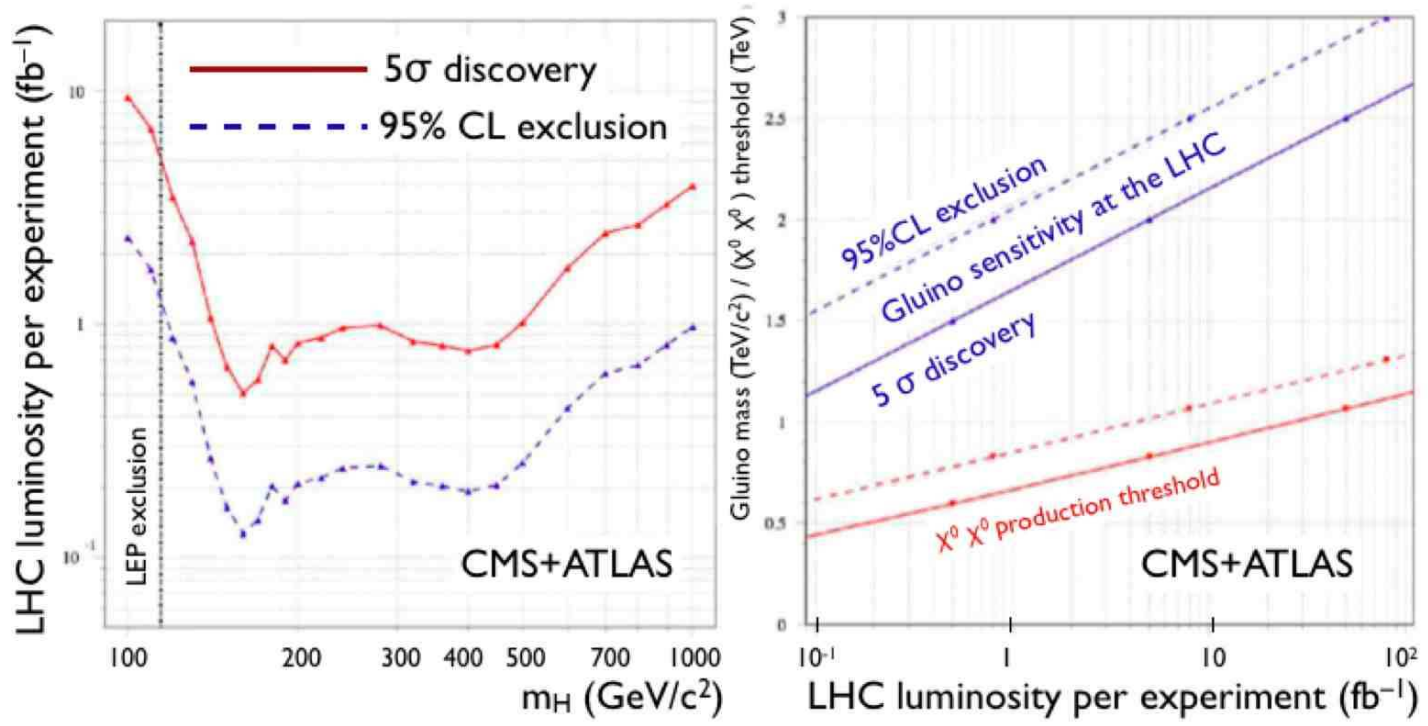

FIGURE 1. The combined sensitivities of ATLAS and CMS to a Standard Model Higgs boson (left), and the gluino (right), as a function of the analyzed LHC luminosity. The right panel also shows the threshold for sparticle pair production at a LC for the corresponding gluino mass, calculated within the CMSSM [3].

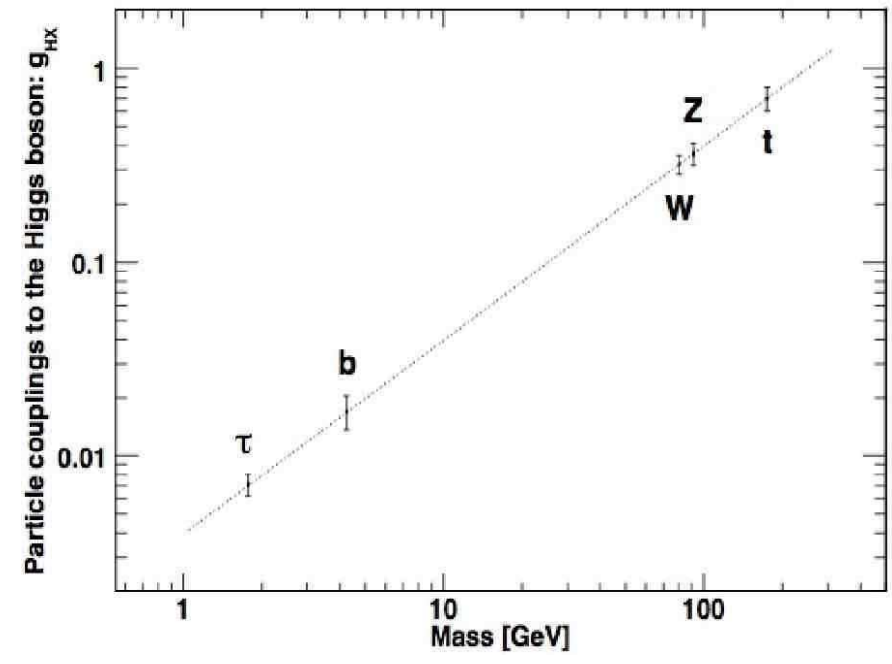

FIGURE 2. Estimates of the accuracy with which experiments at the LHC could measure the couplings of the Higgs boson to various particles [3].

being, the LHC and a possible subsequent LC will be our only direct windows on this physics. 


\section{SUPERSYMMETRY?}

My personal favourite candidate for new physics beyond the Higgs boson is supersymmetry, for several reasons. True, it is intrinsically beautiful and (almost) an essential ingredient in string theory, etc., but these are not the reasons that motivate me to expect that it may appear at the LHC. There are four specific reasons why one might expect supersymmetry to appear around the TeV scale, and hence be accessible to the LHC. One is the naturalness or hierarchy problem [9], another is the unification of the gauge couplings [10], another is the supersymmetric prediction of a light Higgs boson as preferred by the precision electroweak data [11], and another is that many supersymmetric models predict the existence of cold dark matter with a density comparable to that required by astrophysics and cosmology [12].

In the following, I concentrate on the minimal supersymmetric extension of the Standard Model (MSSM). In addition to the coupling $\mu$ between its two gauge multiplets, and the ratio $\tan \beta$ of their vacuum expectation values, the MSSM has many apparently arbitrary soft supersymmetry-breaking parameters, including scalar masses $m_{0}$, gaugino masses $m_{1=2}$, trilinear soft couplings $A_{0}$ and a bilinear soft coupling $B_{0}$. It is commonly assumed that the $m_{0} ; m_{1=2}$ and $A_{0}$ are each universal at some input GUT scale, a framework known as the constrained MSSM (CMSSM). This is not the same as minimal supergravity (mSUGRA), which fixes in addition the gravitino mass: $m_{3=2}=m_{0}$, and imposes $B_{0}=A_{0} \quad m_{0}$. We will see later some potential implications of these extra conditions.

There are direct limits on sparticle masses from their absences at LEP and the Tevatron, and indirect constraints from the LEP lower limit $m_{h}>114 \mathrm{GeV}$ and from $B$ physics, including in particular measurements of $b ! s \gamma$ decay. One possible indication of new physics at the TeV scale may be provided by the BNL measurement of the anomalous magnetic moment of the muon [13], that seems to exhibit a three- $\sigma$ discrepancy with the Standard Model, though this is still somewhat controversial. The strongest constraint on (one combination of) supersymmetric model parameters is provided by the density of cold dark matter: $0: 094<\Omega_{\chi} h^{2}<0: 124$, assuming that it is mainly composed of the lightest neutralino $\chi$.

This not the only possibility: presumably the LSP should have neither strong nor electromagnetic interactions, but there are other candidates that also have these properties. The supersymmetric partners of the neutrinos have been excluded by a combination of LEP and direct dark matter searches, but the LSP might be the spartner of some particle beyond the Standard Model, such as the gravitino.

In a minimal supersymmetric model with universal soft supersymmetry-breaking parameters, one may consider these constraints in the $\left(m_{1=2} ; m_{0}\right)$ plane. As shown in the left panel of Fig. 3, where the LSP is assumed to be the lightest neutralino, the resulting allowed regions include a narrow (pale turquoise) strip near the boundary beyond which $m_{\chi}>m_{\tilde{\tau}_{1}}$ (dark brown shading) where $m_{0} 100 \mathrm{GeV}$, and another strip (not shown) where $m_{0}>1 \mathrm{TeV}$ near the boundary beyond which electroweak symmetry breaking is no longer possible (this region is disfavoured by $g_{\mu} \quad 2$, indicated by pink shading).

The fact that these allowed strips extend to relatively large values of $m_{1=2}$ and $m_{0}$ implies that even the lightest visible sparticles may not be very light, as seen in the right panel of Fig. 3. We generated a large sample of possible CMSSM scenarios (the red 

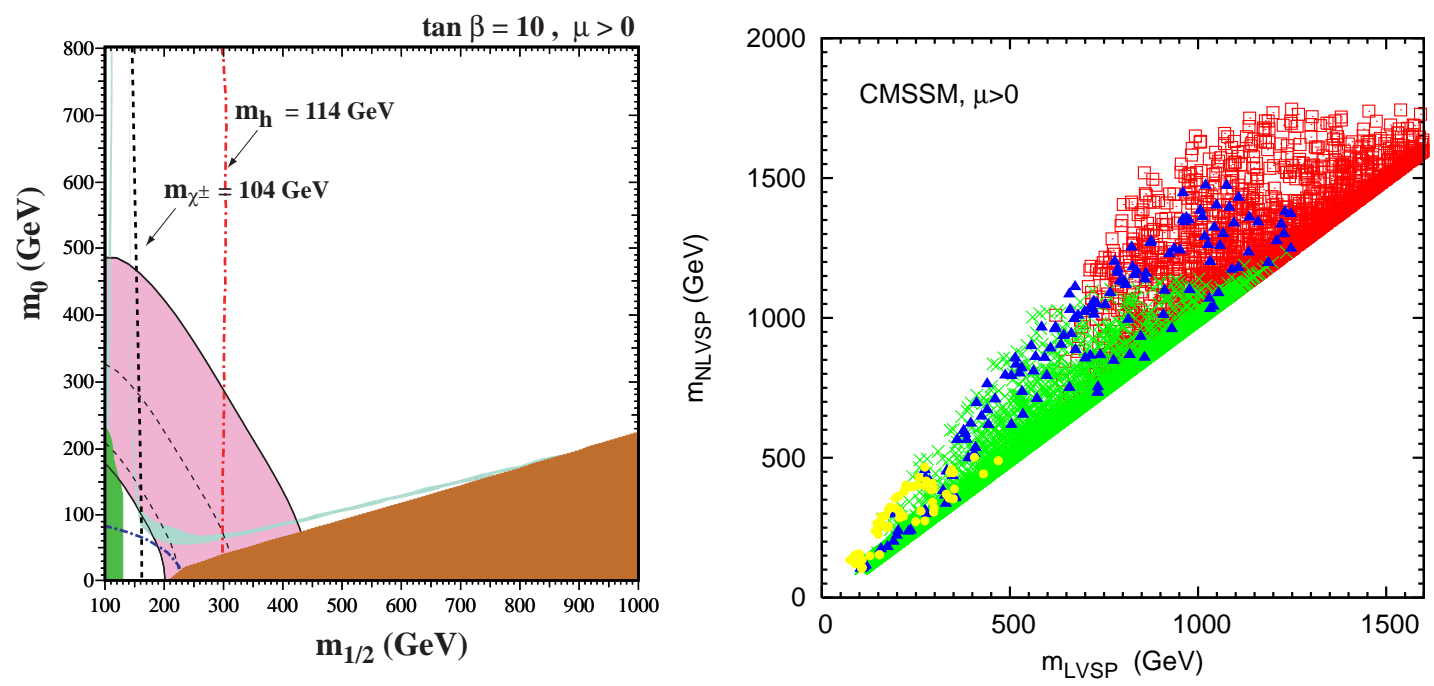

FIGURE 3. The $\left(m_{1=2} ; m_{0}\right)$ plane in the CMSSM for $\tan \beta=10, \mu>0$ and $A_{0}=0$, incorporating the theoretical, experimental and cosmological constraints described in the text (left), and the masses of the lightest and next-to-lightest visible supersymmetric particles in a sampling of CMSSM scenarios (right) [14]. The right panel also indicates the scenarios providing a suitable amount of cold dark matter (blue), those detectable at the LHC (green) and those where the astrophysical dark matter might be detected directly (yellow).

symbols), of which a subsample (shown in darker blue) provide the right amount of cold dark matter [14]. Also shown (in paler green) are the scenarios that would be detectable at the LHC. We see that most (but not all) of the dark matter scenarios should be visible at the LHC: one may be hopeful, but there is no guarantee that the LHC will discover supersymmetry. On the other hand, direct astrophysical dark matter search experiments (very pale yellow) may have less chances in the foreseeable future. An LC with centreof-mass energy $3 \mathrm{TeV}$ would be needed to see sparticles in all the CMSSM dark matter scenarios we sampled.

Can one estimate the scale of supersymmetry on the basis of present data [15]? The precision electroweak measurements of $m_{W}$ and $\sin ^{2} \theta_{W}$ both have some sensitivity to $m_{1=2}$ through radiative corrections, and $m_{W}$ slightly prefers smaller values of $m_{1=2}$, though this trend is hardly significant. The agreement of $b ! s \gamma$ with the Standard Model offers no encouragement to enthusiasts for light supersymmetry, and other $B$-decay observables such as $B_{s} ! \mu^{+} \mu$ and $B_{u}$ ! $\tau \nu$ do not yet provide much information about the possible scale of supersymmetry breaking. On the other hand, the disagreement between the experimental value of $g_{\mu} \quad 2$ with the theoretical value calculated in the Standard Model using low-energy $e^{+} e$ data could be explained by light supersymmetry, as shown already in the left panel of Fig. 3 .

As shown in the left panel of Fig. 44 [15], a global fit to precision electroweak and $B$-decay observables indicates a preference for relatively small values of $m_{1=2}$. This is due predominantly to $g_{\mu} \quad 2$, but there is some support from the measurements of $m_{W}$. Correspondingly, the most likely value for the mass of the lightest supersymmetric Higgs boson is only slightly above the LEP lower limit, as seen in the right panel of Fig. 4 

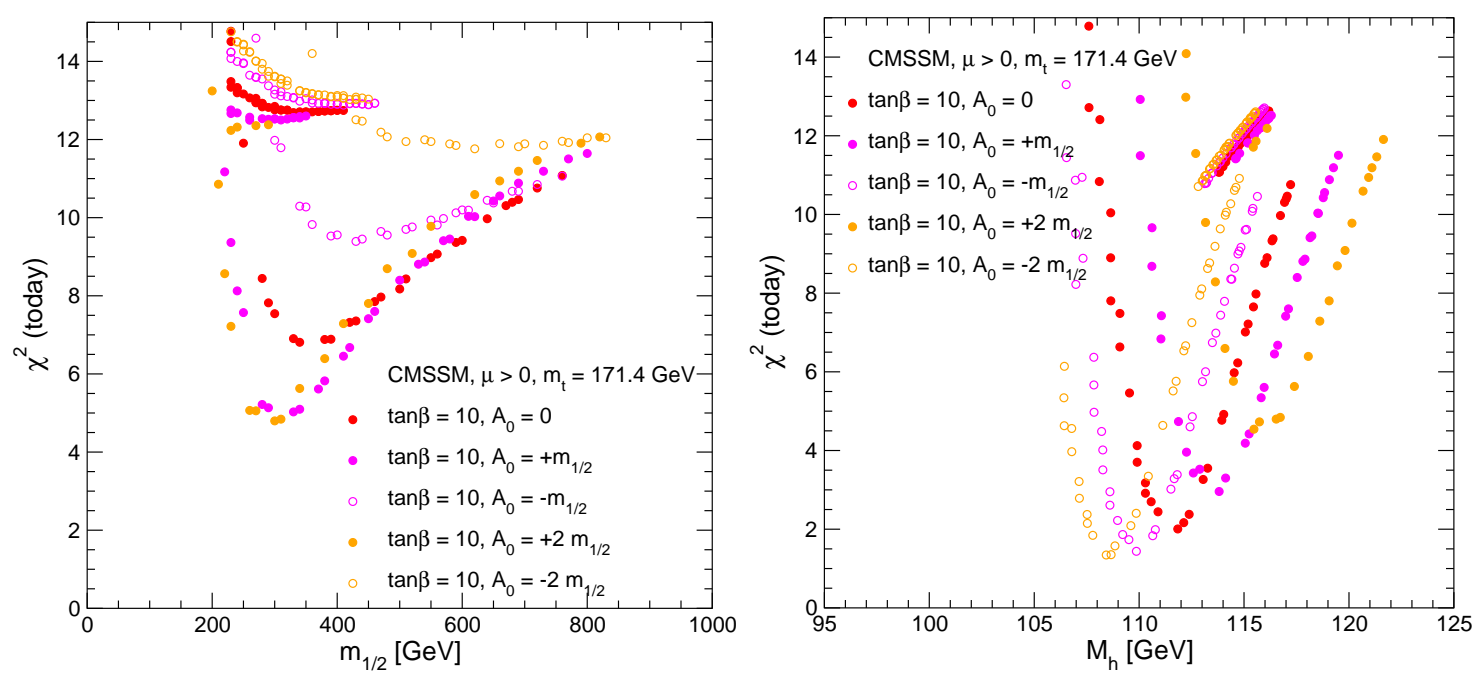

FIGURE 4. Evaluation of $\chi^{2}$ for a global fit to electroweak and $B$-decay observables as a function of $m_{1=2}$ (left) and $m_{h}$ (right) in the CMSSM for $\tan \beta=10, \mu>0$ and different values of $A_{0}$ [15].

The classic signature of sparticle production is the appearance of events with missing energy-momentum carried away by invisible dark matter particles. Studies indicate that such a signature should be observable at the LHC above instrumental and Standard Model backgrounds [1, 2]. In many supersymmetric scenarios, the LHC would produce a pair of squarks and/or gluinos primarily, and these would subsequently decay into a cascade of secondary lighter sparticles, At the end of each decay chain would appear the LSP, namely the lightest neutralino $\chi$ in the CMSSM framework considered so far. Studies indicate that in many benchmark scenarios a number of different sparticle species could be observed at the LHC and their masses measured quite accurately [16, 17]. A LC would produce democratically all the sparticle species that are kinematically accessible, and therefore would be particularly interesting for producing sparticles without strong interactions, such as sleptons, charginos and neutralinos, which are expected to be lighter than squarks and gluinos in CMSSM scenarios.

Even a limited amount of LHC luminosity would enable the LHC to see squarks or gluinos weighing a TeV or more. As shown in the right panel of Fig. 1, it is estimated [1, 2] that $0.1 \mathrm{fb}^{1}$ of LHC luminosity would be sufficient to observe a gluino with a mass of $1.2 \mathrm{GeV}$ at the five- $\sigma$ level, or to exclude a gluino weighing $<15 \mathrm{TeV}$. The discovery and exclusion reaches would extend to about 2.2 and $2.5 \mathrm{TeV}$, respectively with $10 \mathrm{fb} 1$ of LHC luminosity.

As also shown in the right panel of Fig. 1, assuming universal input sparticle masses at the GUT scale, the corresponding thresholds for sparticle pair production at a linear $e^{+} e$ collider would be $0.5(0.6)$ or $0.8(1.0) \mathrm{TeV}[3,18]$. Hence, for example, if the LHC discovers the gluino with $0.1 \mathrm{fb}{ }^{1}$, one may expect that sparticle pair production would be accessible to a linear collider with centre-of-mass energy of $0.5 \mathrm{TeV}$, whereas if the LHC does not discover the gluino even with $10 \mathrm{fb}^{1}$, the $e^{+} e$ sparticle pair production threshold may be above $1 \mathrm{TeV}$. At least in such a simple model, the LHC will tell us how much energy a linear collider would need to find supersymmetry [18]. 
If Nature is kind, and sparticles not only exist but also are quite light, it will be possible to test directly unification of the gauge couplings and universality of the soft supersymmetry-breaking scalar masses with high precision, in particular by comparing measurements at the LHC and the ILC [19]. However, there is no guarantee that any sparticles will be light enough to be produced at the ILC, even with a centre-of-mass energy of $1 \mathrm{TeV}$. For this reason, and also because even if there are some light sparticles the heavier ones will be produced only at higher energies, a high-energy LC would be advantageous, which is why CERN and its collaborators are developing the CLIC technology that should be capable of reaching $3 \mathrm{TeV}$ in the centre of mass [20].

So far, I have concentrated on the CMSSM, assuming that all the soft supersymmetrybreaking scalar masses are universal at the GUT scale. However, this may not be the case: in particular, there is no good theoretical or phenomenological reason why the Higgs scalar masses should be universal. In models with non-universal Higgs masses (NUHM), there are two additional degrees of freedom, and the Higgs mixing parameter $\mu$ and the pseudoscalar Higgs mass $m_{A}$ may be treated as free parameters [21].

We have studied [22] whether the NUHM framework could accommodate a pseudoscalar supersymmetric Higgs boson as light as the value not yet excluded by direct searches at the Tevatron. Recall that the CDF experiment saw a two- $\sigma$ excess in the $\tau^{+} \tau$ spectrum that could be explained by supersymmetric Higgs bosons weighing

$160 \mathrm{GeV}$ [23], whereas the D0 experiment saw no such excess [24]. We found [22] that $m_{A} \quad 160 \mathrm{GeV}$ could indeed be accommodated within the NUHM, if $\tan \beta \quad 50 ; m_{1=2}$ $600 \mathrm{GeV}, m_{0} \quad 800 \mathrm{GeV}, \mu \quad 400 \mathrm{GeV}$ and $A_{0} \quad 2 \mathrm{TeV}$, as seen in Fig. 5, This would be quite an extreme scenario, but it would have the merit of being testable in the near future. In this corner of the NUHM parameter space, $m_{h} ; b ! s \gamma_{;} B_{s} ! \mu^{+} \mu \quad ; B_{u}$ ! $\tau v$ and the cold dark matter scattering rates would all be very close to the present experimental limits.

\section{GRAVITINO DARK MATTER?}

The LSP should have no strong or electromagnetic interactions, otherwise it would bind to conventional matter and be detectable as some anomalous heavy nucleus. Apart from the lightest neutralino $\chi$, possible weakly-interacting scandidates include the sneutrino, which is excluded by LEP and direct searches in simple models, and the gravitino $\tilde{G}$, which has attracted relatively little detailed attention until recently. It would be a nightmare for astrophysical detection, but a bonanza for the LHC [25, 26, 27], as we now discuss.

The next-to-lightest sparticle (NLSP) would have a very long lifetime in models with gravity-mediated supersymmetry breaking, due to the weak gravitational strength of the interactions responsible for its decays. For example, if the NLSP is the lighter stau slepton $\tilde{\tau}_{1}$ one has

$$
\Gamma_{\tilde{\tau}_{1} ! \tau \tilde{G}}=\frac{1}{48 \pi} \frac{1}{M_{P}^{2}} \frac{m_{\tilde{\tau}_{1}}^{5}}{m_{\tilde{G}}^{2}} 1 \frac{m_{\tilde{G}}^{2}}{m_{\tilde{\tau}_{1}}^{2}} ;
$$

leading to a lifetime that could be measured in hours, days, weeks, months or even years! The stau is not the only candidate for the NLSP. Other generic possibilities include the 

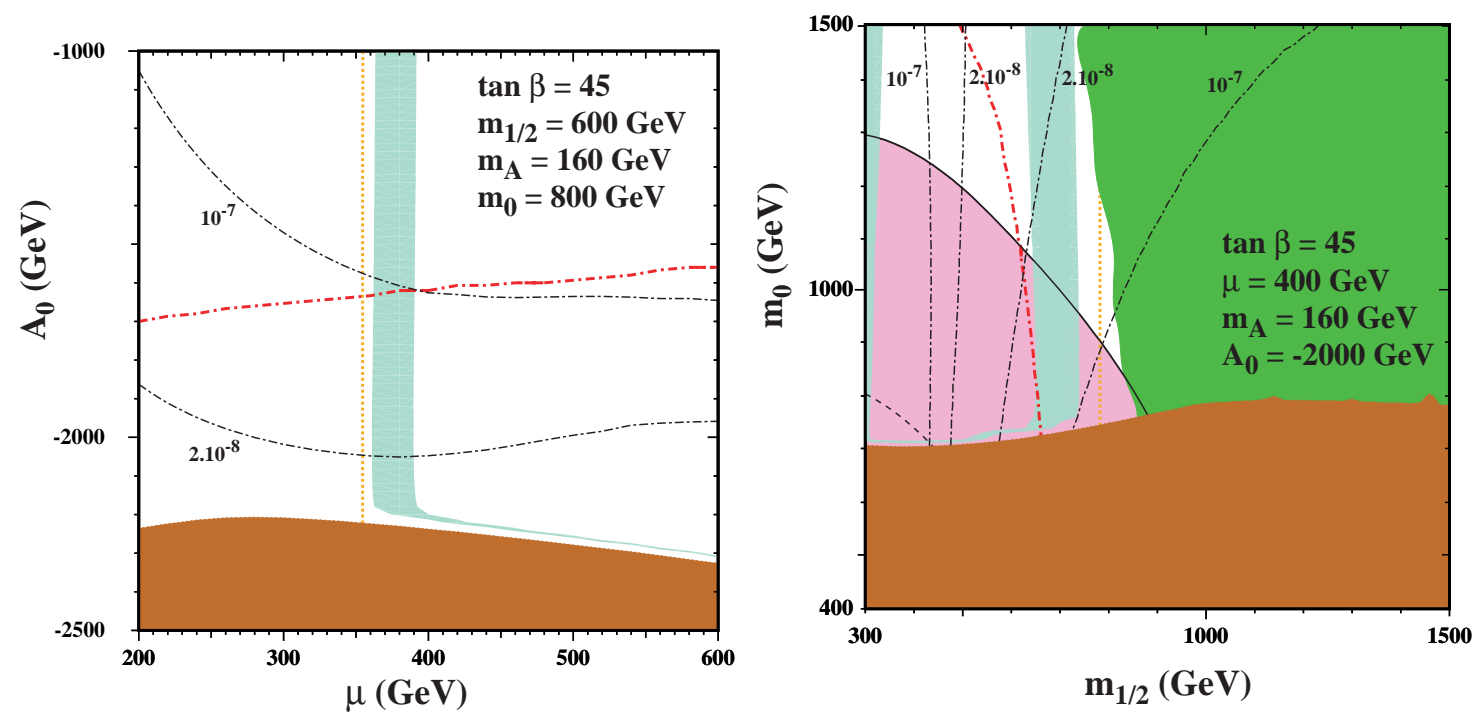

FIGURE 5. A light $m_{A} \quad 160 \mathrm{GeV}$ could be accommodated within the NUHM, if $\tan \beta \quad 50 ; m_{1=2}$ $600 \mathrm{GeV}, m_{0} \quad 800 \mathrm{GeV}, \mu \quad 400 \mathrm{GeV}$ and $A_{0} \quad 2 \mathrm{TeV}$, in which case $m_{h} ; b ! s \gamma ; B_{s} ! \mu^{+} \mu \quad ; B_{u} ! \tau \nu$ and the cold dark matter scattering rates would all be very close to the present experimental limits [22].

lightest neutralino $\chi$, a sneutrino [28], or even the lighter stop squark [29]. In the case of minimal supergravity (mSUGRA), one finds that a gravitino LSP combined with a stau NLSP is as generic as the conventional neutralino LSP scenario, as seen by the light (yellow) shaded region in the left panel of Fig. 6 All of these possibilities are constrained by astrophysics and cosmology, particularly through limits on the decays of the metastable NLSP [30], and its bound states if it is charged [31]. These effects may even improve the agreement between cosmological nucleosynthesis calculations and the observed Lithium abundances, as shown in the pink (darker) shaded region in the right panel of Fig. 6 for one particular non-mSUGRA scenario [32]. This scenario could be probed only with a higher-energy LC [33].

We recently examined how a stau NLSP scenario could be explored at the LHC [25, 26, 27]. We found that the normal experimental triggers on jets and energetic muons and electrons would select the events containing staus [26, 27]. It would then be possible to identify the stau tracks in the events with quite high efficiency, and measure the stau mass very accurately via a combination of momentum and time-of-flight measurements, as seen in Fig. 7. The stau could be combined with jets in the event to reconstruct the masses of heavier sparticles in the supersymmetric decay cascades, as in neutralino LSP scenarios, as shown in Fig 8

Very slow-moving staus might be stopped either in the detector material or in the rock surrounding the cavern [34, 35]. One could use the muon system to locate the stau's impact point on the cavern wall with an uncertainty $1 \mathrm{~cm}$, and its impact angle with an accuracy $10^{3}$. One might then be able to bore into the cavern wall and remove a core from the rock, which one could then store while waiting for the stau to decay. Before boring, one would have to wait for a shut-down of the LHC, since the radioactivity in the cavern is quite high while it is operating. However, it is planned to stop the accelerator 

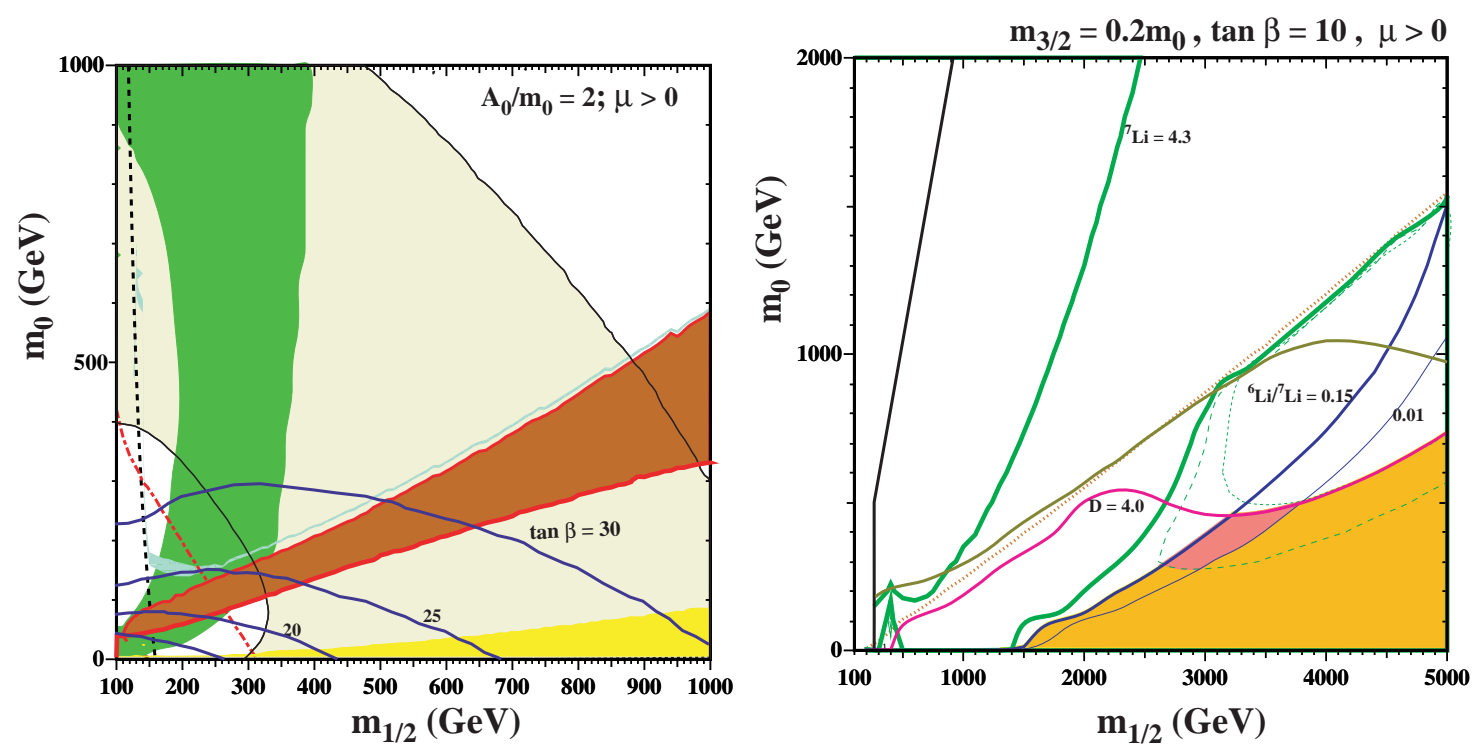

FIGURE 6. In the mSUGRA scenario with $A_{0}=2 m_{0}$ and $\mu>0$ (left panel), in addition to the allowed $\chi$ dark matter strip (light turquoise) and the forbidden $\tilde{\tau}_{1}$ LSP wedge (dark brown), there is a wedge (pale yellow) with a metastable $\tilde{\tau}_{1}$ whose decays do not disturb the cosmological light element abundances. In the non-SUGRA scenario with $m_{\tilde{G}}=02 m_{0}$ (right panel), there is also a region (darker pink) where $\tilde{\tau}_{1}$ bound-state effects [31] may improve the Lithium abundances [32].
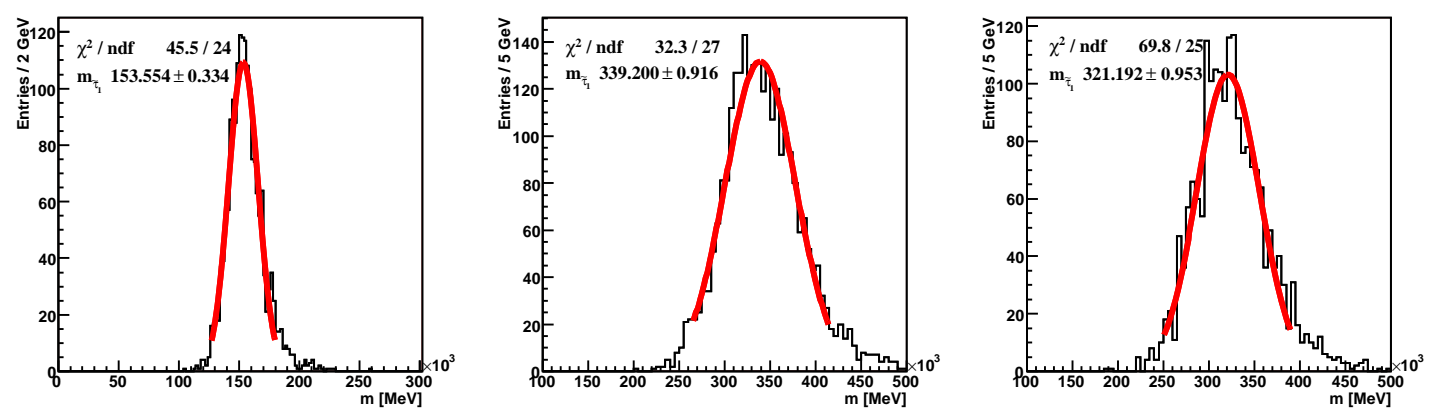

FIGURE 7. The mass of a metastable stau could be measured quite accurately at the LHC [27], as exemplified in three benchmark scenarios [25].

for a couple of days every month, so this strategy might work if the lifetime exceeds about $10^{6} \mathrm{~s}$ !

\section{EXTRA DIMENSIONS?}

Supersymmetry is certainly not the only possibility for new physics at the LHC. Another possibility offered by string theory is that there might be large extra dimensions. When string theory was originally proposed as a 'Theory of Everything', it was imagined that all the extra dimensions would be curled up on length scales comparable to the 

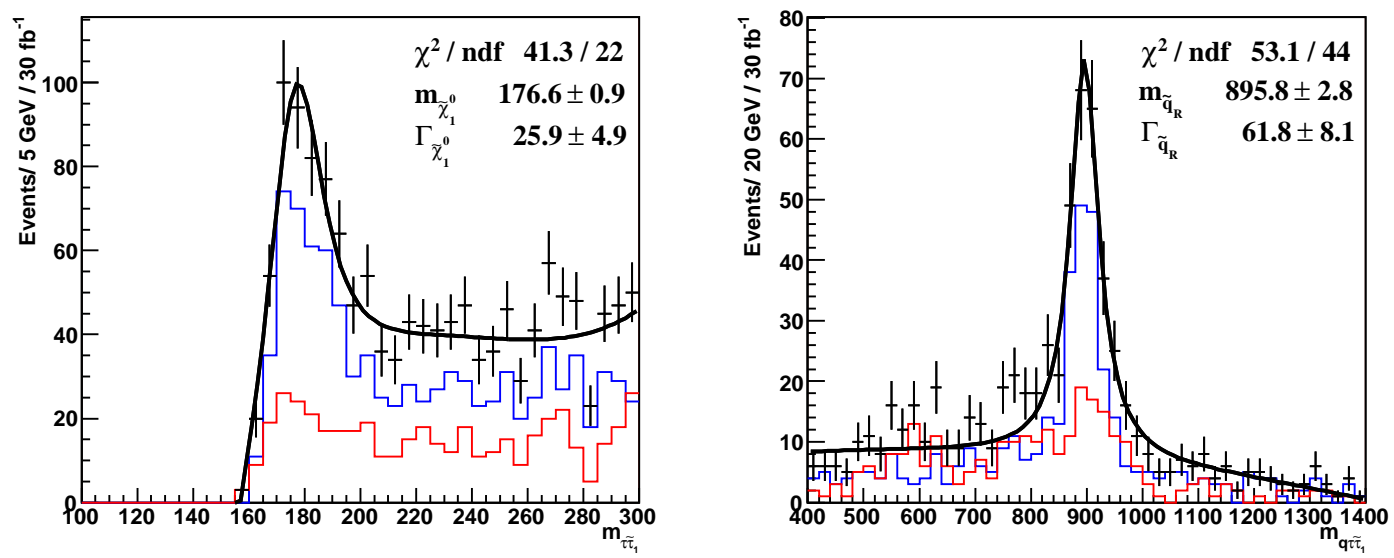

FIGURE 8. The reconstruction of heavier sparticles decaying into the $\tilde{\tau}$ in a scenario where it is the NLSP and the gravitino is the LSP [26].

Planck length $10^{33} \mathrm{~cm}$. However, then it was realized that string unification could be achieved more easily if one of these dimensions was somewhat smaller than the GUT scale [36], and a number of scenarios with much larger extra dimensions have been considered. For example, an extra dimension of size $1 \mathrm{TeV}{ }^{1}$ could help break supersymmetry [37] and/or the electroweak gauge symmetry, an extra dimension of micron size could help rewrite the hierarchy problem [38], and even infinite extra dimensions are allowed if they are warped appropriately [39].

In many of these scenarios, there are potential signals to be found at the LHC, such as Kaluza-Klein excitations of gravitons, or missing energy 'leaking' into an extra dimension. The most spectacular possibility would occur if gravity becomes strong at the $\mathrm{TeV}$ energy scale, in which case microscopic black holes might be produced at the LHC. These would be very unstable, decaying rapidly via Hawking radiation into multiple jets, leptons and photons, that would be easily detectable [40] and distinguishable from supersymmetry and other extra-dimensional scenarios [41].

\section{SUMMARY}

The origin of mass is the most pressing problem in particle physics, and requires a solution within the LHC energy range. Will it be a simple Higgs boson? and/or supersymmetry? The LHC will tell! there are many speculative ideas for other possible new physics beyond the Standard Model, such as grand unification, strings, and extra dimensions. The LHC is also capable of probing many of these speculations including, as the last two examples show, novel ideas that were undreamt of when the LHC and its experiments were designed. We do not know what the LHC will find, but we can be sure that its discoveries will set the agenda for possible future projects, such as a linear $e^{+} e$ collider. 


\section{REFERENCES}

1. ATLAS Collaboration, Detector and Physics Performance Technical Design Report, LHCC 99-14/15 (1999).

2. CMS Collaboration, CMS Physics Technical Design Report. Volume II: Physics Performance, CERN/LHCC 2006-021, CMS TDR 8.2 (2006).

3. A. Blondel, L. Camilleri, A. Ceccucci, J. Ellis, M. Lindroos, M. Mangano and G. Rolandi, Physics opportunities with future proton accelerators at CERN, arXiv:hep-ph/0609102

4. S. Y. Choi, D. J. Miller, M. M. Muhlleitner and P. M. Zerwas, Phys. Lett. B 553 (2003) 61 [arXiv:hep-ph/0210077].

5. C. P. Buszello, I. Fleck, P. Marquard and J. J. van der Bij, Eur. Phys. J. C 32 (2004) 209 [arXiv:hep-ph/0212396].

6. E. Accomando et al., Workshop on CP studies and non-standard Higgs physics, arXiv:hep-ph/0608079.

7. D. J. Miller, S. Y. Choi, B. Eberle, M. M. Muhlleitner and P. M. Zerwas, Phys. Lett. B 505 (2001) 149 [arXiv:hep-ph/0102023].

8. G. Weiglein et al. [LHC/LC Study Group], Phys. Rept. 426 (2006) 47 [arXiv:hep-ph/0410364]; A. Djouadi, J. Lykken, K. Monig, Y. Okada, M. J. Oreglia and S. Yamashita et al., International Linear Collider Reference Design Report Volume 2: Physics at the ILC, arXiv:0709.1893 [hep-ph].

9. L. Maiani, All You Need To Know About The Higgs Boson, Proceedings of the Gif-sur-Yvette Summer School On Particle Physics, 1979, pp.1-52; G. 't Hooft, in Recent developments in Gauge Theories, Proceedings of the NATO Advanced Study Institute, Cargèse, 1979, eds. G. 't Hooft et al. (Plenum Press, NY, 1980); E. Witten, Phys. Lett. B 105 (1981) 267.

10. J. R. Ellis, S. Kelley and D. V. Nanopoulos, Phys. Lett. B 249 (1990) 441 and Phys. Lett. B 260 (1991) 131; U. Amaldi, W. de Boer and H. Furstenau, Phys. Lett. B 260 (1991) 447; C. Giunti, C. W. Kim and U. W. Lee, Mod. Phys. Lett. A 6 (1991) 1745.

11. M. W. Grunewald, arXiv:0709.3744 [hep-ex].

12. J. R. Ellis, J. S. Hagelin, D. V. Nanopoulos, K. A. Olive and M. Srednicki, Nucl. Phys. B 238 (1984) 453.

13. H. N. Brown et al. [Muon g-2 Collaboration], Phys. Rev. Lett. 86 (2001) 2227 [arXiv:hep-ex/0102017].

14. J. R. Ellis, K. A. Olive, Y. Santoso and V. C. Spanos, Phys. Lett. B 603 (2004) 51 [arXiv:hep-ph/0408118].

15. J. R. Ellis, S. Heinemeyer, K. A. Olive, A. M. Weber and G. Weiglein, arXiv:0706.0652 [hep-ph].

16. I. Hinchliffe, F. E. Paige, M. D. Shapiro, J. Soderqvist and W. Yao, Phys. Rev. D 55 (1997) 5520 [arXiv:hep-ph/9610544].

17. M. Battaglia, A. De Roeck, J. R. Ellis, F. Gianotti, K. A. Olive and L. Pape, Eur. Phys. J. C 33 (2004) 273 [arXiv:hep-ph/0306219]; A. De Roeck, J. R. Ellis, F. Gianotti, F. Moortgat, K. A. Olive and L. Pape, arXiv:hep-ph/0508198

18. This discussion is being extended in J. R. Ellis, K. A. Olive and P. Sandick, in preparation.

19. P. Bechtle, K. Desch, W. Porod and P. Wienemann, Eur. Phys. J. C 46 (2006) 533 [arXiv:hep-ph/0511006].

20. E. Accomando et al. [CLIC Physics Working Group], Physics at the CLIC multi-TeV linear collider, arXiv:hep-ph/0412251.

21. J. Ellis, T. Falk, K. Olive and Y. Santoso, Nucl. Phys. B 652 (2003) 259, hep-ph/0210205

22. J. R. Ellis, S. Heinemeyer, K. A. Olive and G. Weiglein, Phys. Lett. B 653 (2007) 292 arXiv:0706.0977 [hep-ph].

23. CDF Collaboration, CDF note 8676 , see: http://www-cdf.fnal.gov/ aa/mssm_htt_1fb/note/cdf8676.pdf

24. D0 Collaboration, D0 Note 5331-CONF, see: http://www-d0.fnal.gov/cgi-bin/d0note?5331.

25. A. De Roeck, J. R. Ellis, F. Gianotti, F. Moortgat, K. A. Olive and L. Pape, Eur. Phys. J. C 49 (2007) 1041 [arXiv:hep-ph/0508198].

26. J. R. Ellis, A. R. Raklev and O. K. Oye, JHEP 0610 (2006) 061 [arXiv:hep-ph/0607261].

27. J. R. Ellis, A. R. Raklev and O. K. Oye, in preparation.

28. L. Covi and S. Kraml, arXiv:hep-ph/0703130 
29. J. L. Diaz-Cruz, J. R. Ellis, K. A. Olive and Y. Santoso, JHEP 0705 (2007) 003 |arXiv:hep-ph/0701229].

30. R. H. Cyburt, J. R. Ellis, B. D. Fields and K. A. Olive, Phys. Rev. D 67 (2003) 103521 [arXiv:astro-ph/0211258].

31. M. Pospelov, Phys. Rev. Lett. 98 (2007) 231301 [arXiv:hep-ph/0605215].

32. R. H. Cyburt, J. R. Ellis, B. D. Fields, K. A. Olive and V. C. Spanos, JCAP 0611 (2006) 014 [arXiv:astro-ph/0608562].

33. O. Cakir, I. T. Cakir, J. R. Ellis and Z. Kirca, arXiv:hep-ph/0703121

34. J. L. Feng and B. T. Smith, Phys. Rev. D 71 (2005) 015004 [Erratum-ibid. D 71 (2005) 0109904] [arXiv:hep-ph/0409278].

35. K. Hamaguchi, Y. Kuno, T. Nakaya and M. M. Nojiri, Phys. Rev. D 70 (2004) 115007 [arXiv:hep-ph/0409248].

36. E. Witten, Nucl. Phys. B 471 (1996) 135 [arXiv:hep-th/9602070].

37. I. Antoniadis, Phys. Lett. B 246 (1990) 377.

38. I. Antoniadis, N. Arkani-Hamed, S. Dimopoulos and G. R. Dvali, Phys. Lett. B 436 (1998) 257 [arXiv:hep-ph/9804398].

39. L. Randall and R. Sundrum, Phys. Rev. Lett. 83 (1999) 3370 [arXiv:hep-ph/9905221].

40. C. M. Harris, M. J. Palmer, M. A. Parker, P. Richardson, A. Sabetfakhri and B. R. Webber, JHEP 0505 (2005) 053 [arXiv:hep-ph/0411022].

41. J. M. Smillie and B. R. Webber, JHEP 0510 (2005) 069 [arXiv:hep-ph/0507170]. 